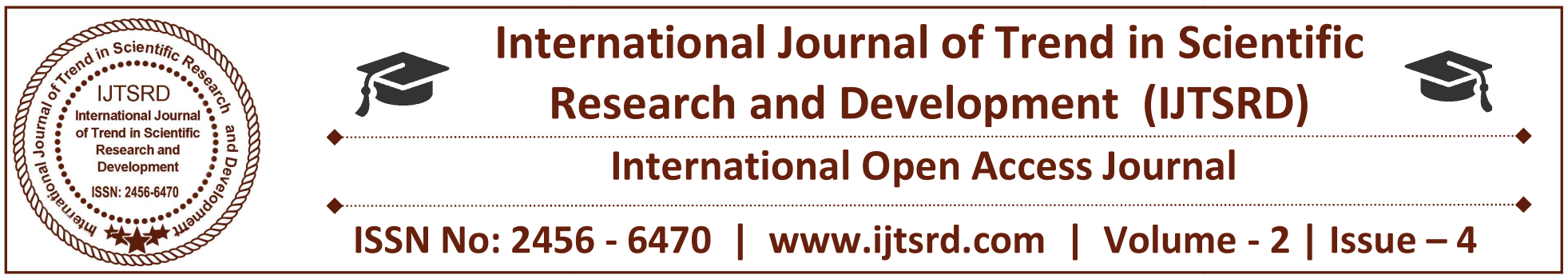

\title{
A Novel Approach for Progressive Duplicate Detection for Quality Assurance
}

\author{
S. Divya \\ MCA Final Year, Lakireddy Balireddy College of Engineering, \\ Mylavaram, Andra Pradesh, India
}

\begin{abstract}
In reality the data set may have at least one portrayal of a similar certifiable elements. Duplicate may emerge because of exchange errors and because of deficient information. Expelling such duplicate, all things considered, is a perplexing errand. It isn't direct to productively discover and expel the duplicates from a vast data set. This paper center around correlation with conventional duplicate discovery strategies Incremental Sorted Neighborhood Method (ISNM) and the Duplicate Count Strategy $(\mathrm{DCS}++)$ technique with Progressive Sorted Neighborhood Method (PSNM) technique.
\end{abstract}

Keywords: Duplicate Detection, Dataset, Duplicate Count Strategy

\section{INTRODUCTION}

Databases are vital part of an organization, for example, the greater part of its information in kept in it, influencing the information to set exceptional without duplicates and to keep its information purifying the duplicate location assumes an imperative part. For keeping the nature of information inside the organization it's tedious and expensive. The greater part of the current framework faces the issue of discovering duplicates prior in the recognition procedure. Element determination strategy [1] distinguishes the various portrayal of same character. The dynamic Sorted Neighborhood strategy [2] lessens the normal time for which duplicate is found. Be that as it may, the current strategies Incremental Sorted Neighborhood Method [5] finds the duplicates in a given window and duplicate check strategy [3], finds the duplicate by expanding the window estimate by the quantity of duplicates identified.

\section{Incremental Duplicate Detection Method}

This Method is the augmentation of the essential Sorted Neighborhood Method. In this technique at first it sorts the given data set utilizing choice sort in view of an arranging key. Arranging is performed so that the comparative tuples are near each other. The arranging key is extraordinary and isn't virtual at that point characterizes the window measure at that point thinks about the records inside that window indicated.

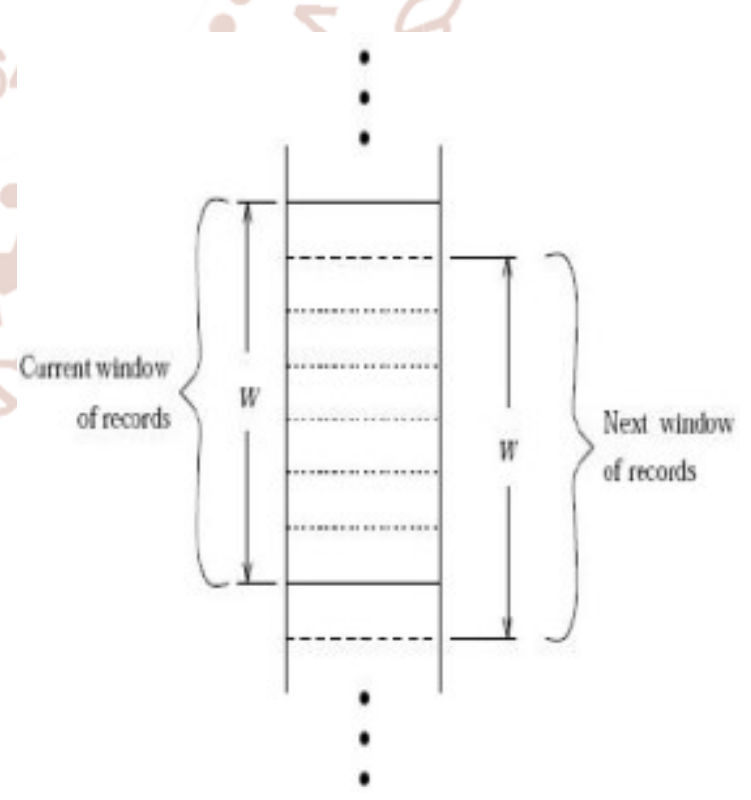

Fig. 1: Window Sliding Example 
Fig. 1 shows how the predefined window measure slides over the given instructive record. There are heaps of connections in this technique recollecting a definitive goal to decrease the examination and to discover more duplicates inside the predefined window Duplicate Count Strategy ++ is utilized which broadens the window assess in context of the measure of duplicates perceived.

\section{Duplicate Count Strategy ++}

This method is the expansion of DCS Duplicate Count Strategy. It is a system which progressively modifies the window measure. That is it differentiates the window measure in light of the measure of duplicates apparent. Change will now and again expansion or decreasing the measure of connections If more duplicates of a record are found inside a window, the more noteworthy the window ought to be If no such duplicate of a record inside its neighborhood is found, expect that there are no duplicates or the duplicates are exceptionally far away in the organizing request.

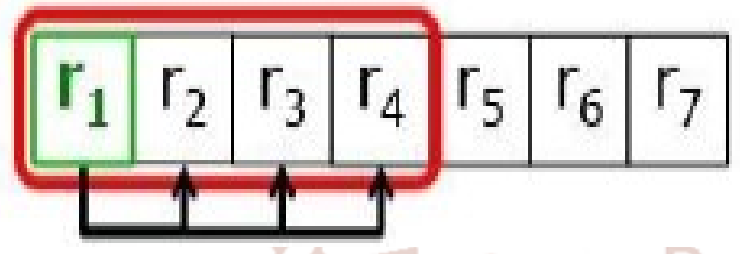

Fig. 2. Comparing records in DCS++

Each tuple ti is once toward the start of a window $w$ it is then Compare with $\mathrm{w} 1$ successors If no duplicate for ti is discovered, proceed as regular else increment window. DCS+ for finding amazing source is delineates as tails:

- Sorts the given enlightening gathering

- demonstrate the window w

- Compare the fundamental record in the window with that of a large portion of the records in the window which is appeared in Fig.2

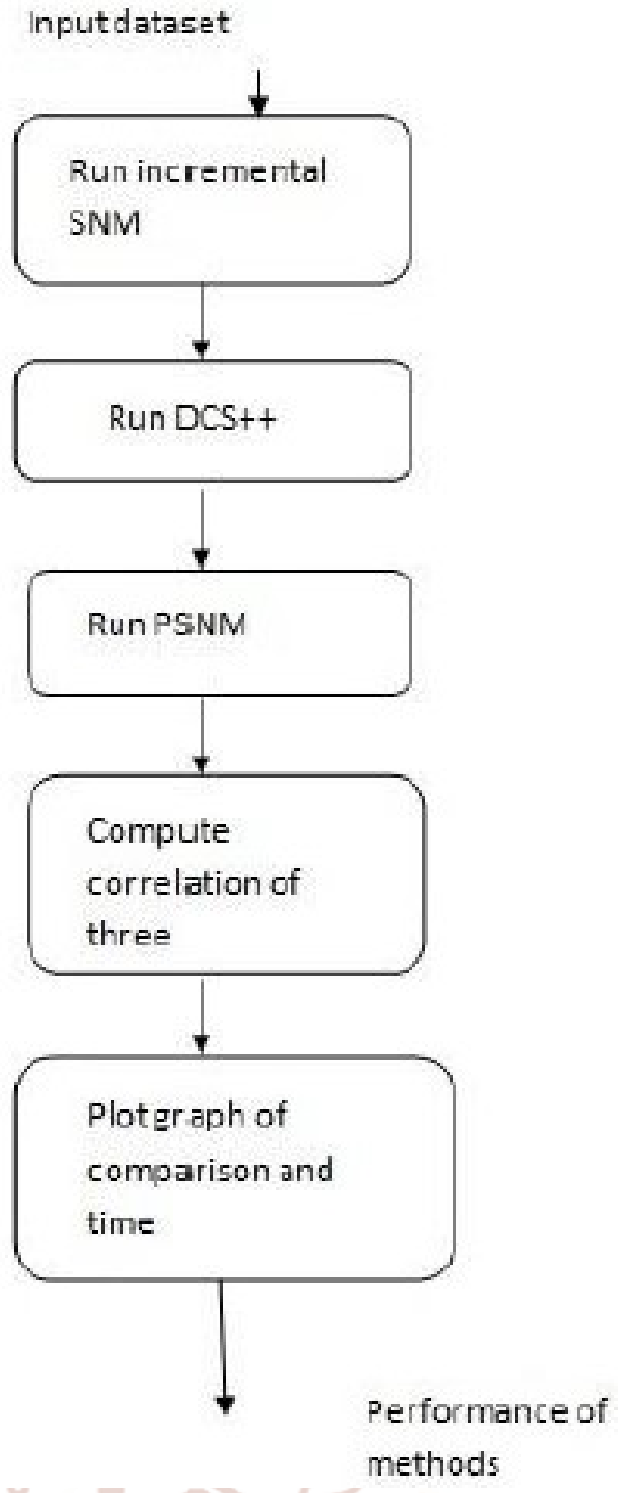

Fig. 3. Flow Chart for performing the detection

- Increment window measure while duplicate perceived/examination $\geq \varphi$ where $\varphi$ is a state of control

- Slide the window if no duplicates found inside the window

- If duplicates found, for each perceived duplicate the going with w-1 records of that duplicate are added to the window.

- Duplicates are perceived.

The Fig. 3 above displays to play out these duplicate unmistakable evidence techniques

\section{Dynamic Sorted Neighborhood Method}

This strategy sensibly finds the duplicate. At first it sorts the information and portrays a window survey it 
packages the whole instructive rundown in light of the piece size and looks records inside the window appeared in each bit. With a specific extreme target to reliably discover the duplicates the PSNM count depicts an extension between times. The expansion between time shifts from the humblest window size to the most unprecedented that is w-1. Thusly guaranteeing the promising close neighbors are picked first and less consoling records later. The PSNM computation manufactures the ability of discovering duplicates by seriously changing the window assess. In the present framework there is an issue stack the illuminating record each opportunity to look at however in this procedure it stack the segment once and by changing the advancement break it effectively perceive the duplicates.

\section{RESULT}

Contrasted with the two frameworks used as a piece of copy recognizable proof, ISNM and DCS++ technique simply find copy. It isn't much profitable. At first the copies are not found, simply less connections and less number of the duplicate perceived.

1) Duplicate Detection Method which is "fit and financially savvy

2) It reports the duplicates prior in the zone framework

3) Windowing thought is utilized for finding the measure of duplicates. So the PSNM is the basic framework to manage current divulgence issues.

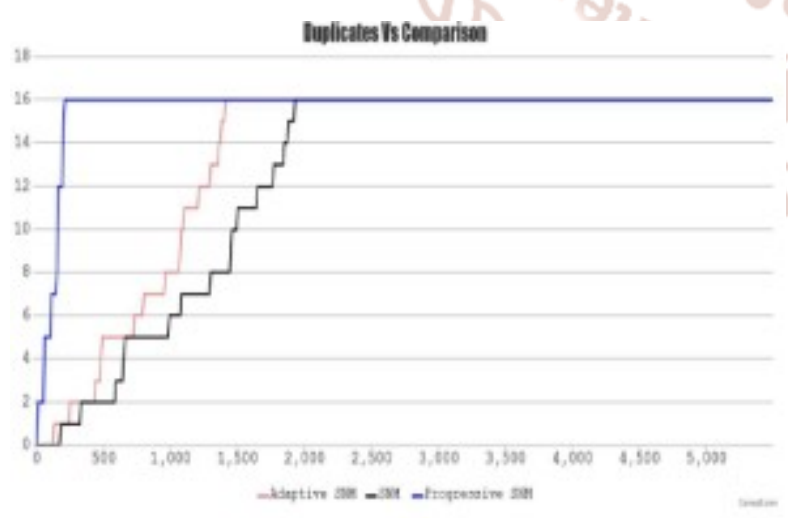

Fig. 4. Analysis based on number of comparison

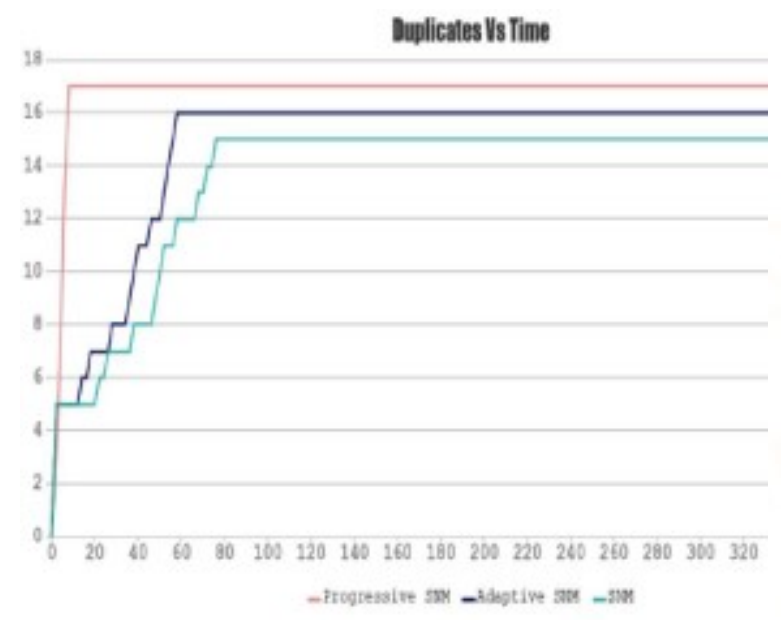

Fig. 5. Analysis based on time taken

Table 1: Comparison Table

\begin{tabular}{|c|c|l|}
\hline S.N0: & IIETHOD & \multicolumn{1}{|c|}{ FWNCTION } \\
\hline 1 & ISNM & $\begin{array}{l}\text { - No widowing Concept } \\
\text { - Less efficient }\end{array}$ \\
\hline 2 & DCSH & $\begin{array}{l}\text { - Windorw size increases based on the number of the } \\
\text { duplicates detected }\end{array}$ \\
\hline 3 & PSNM & $\begin{array}{l}\text { - Progressive Techinique } \\
\text { - More efficient }\end{array}$ \\
\hline
\end{tabular}

\section{CONCLUSION}

Dynamic Duplicate Detection Method help to produce

report amount of copy found in the educational file. Along these lines this methodology tries ti improves the typical time between the copies distinguishing proof. PSNM calculation constantly run the calculation by the essentially picking the customer demonstrated parameters, for instance, window measure, part measure and whatnot when we endeavor to execute this productive figuring as a web application it will require more noticeable dare to discover duplicates. Hadoop Map lessening can be utilized to overhaul the sufficiency by giving the key and the check of duplicate perceived.

\section{REFERENCES}

1. O. Hassanzadeh, R. J. Miller, "Creating probabilistic databasesfrom duplicated data," VLDB J., vol. 18, no. 5, pp. 1141-1166, 2009.

2. U. Draisbach, F. Naumann, S. Szott, O. Wonneberg,"Adaptive windows for duplicate detection," in Proc. IEEE $28^{\text {th }}$ Int. Conf. Data Eng., 2012, pp. 1073-1083.

3. S. Yan, D. Lee, M.-Y. Kan, L. C. Giles, “Adaptive sorted neighborhood methods efficient record 
linkage," in Proc. 7th ACM/IEEE Joint Int. Conf. Digit. Libraries, 2007, pp. 185-194.

4. J. Madhavan, S. R. Jeffery, S. Cohen, X. Dong, D. Ko, C. Yu,A. Halevy, "Web-scale data integration: You can onlyafford to pay you go," in Proc. Conf. Innovative Data Syst.Res., 2007.

5. Progressive Duplicate Detection Thorste Papenbrock, Arvid Heise, Felix Naumann in 2015

6. S. E. Whang, D. Marmaros, H. Garcia-Molina, Pay-as-you- entity resolution, IEEE Trans. Knowl. Data Eng., vol. 25, no. 5, May 2012

7. M. A. Hernandez S. J. Stolfo, Real-world data is dirty: Data cleansing and the merge/purge problem, Data Mining Knowl. Discovery, vol. 2, no. 1, pp. 937,1998

8. U. Draisbach and F. Naumann, "A generalization of blocking andwindowing algorithms for duplicate detection," Proc. Int. Conf.Data Knowl. Eng., 2011, pp. 18-24.

9. H. S. Warren, "A modification of Warshall's algorithm for thetransitive closure of binary relations," Commun. ACM, vol. 18,no. 4, pp. 218-220, 1975.

10. M. Wallace, S. Kollias, "Computationally efficient incrementaltransitive closure of sparse fuzzy binary relations," in Proc. IEEEInt. Conf. Fuzzy Syst., 2004, pp. 1561-1565.

11. F. J. Damerau, "A technique for computer detection, correction of spelling errors," Commun. ACM, vol. 7, no. 3, pp. 171-176,1964.

12. P. Christen, "A survey of indexing techniques for scalable recordlinkage deduplication," IEEE
Trans. Knowl. Data Eng., vol. 24,no. 9, pp. 15371555, Sep. 2012.

13. B. Kille, F. Hopfgartner, T. Brodt, T. Heintz, "The Plista dataset," in Proc. Int. Workshop Challenge News Recommender Syst., 2013,pp. 16-23.

14. Priyanka et al., International Journal of Advanced Research in Computer Science and Software Engineering 6(8), August- 2016, pp. 332-335

15. U. Draisbach, F. Naumann, A generalization of blocking, windowing algorithms for duplicate detection, in Proc. Int. Conf. Data Knowl. Eng., 2011, pp. 1824.

16. S. Yan, D. Lee, M.-Y. Kan, L. C. Giles, Adaptive sorted neighborhood methods for efficient record linkage, in Proc. 7th ACM/ IEEE Joint. Conf. Digit. Libraries, 2007, pp. 185194.

17. U. Draisbach, F. Naumann, S. Szott, O. Wonneberg, Adaptive windows for duplicate detection, in Proc. IEEE 28th Int. Conf. Data Eng., 2012, pp. 10731083.

18. L. Kolb, A. Thor, E. Rahm, "Parallel sorted neighborhood blocking with MapReduce," in Proc. Conf. Datenbanksysteme Buro, Technik und Wissenschaft $€, 2011$.

19. M. Wallace and S. Kollias, "Computationally efficient incrementaltransitive closure of sparse fuzzy binary relations," in Proc. IEEEInt. Conf. Fuzzy Syst., 2004, pp. 1561-1565.

20. F. J. Damerau, "A technique for computer detection and correctionof spelling errors," Commun. ACM, vol. 7, no. 3, pp. 171-176,1964. 